\title{
Experimental Evaluation of Human Grasps Using a Sensorized Object
}

\author{
Maximo A. Roa, Risto Kõiva and Claudio Castellini
}

\begin{abstract}
Grasp quality measures have been studied for long time, given their importance to evaluate the goodness/convenience of a grasp made with a robotic hand. However, the application of these quality measures to grasps made by humans has just recently received some attention. This paper presents an experimental evaluation and comparison of different measures, using data obtained with a sensorized object. The experiment compares power grasps and precision grasps obtained with different number of fingers. The results intend to be a guide to the application of such qualities in the evaluation of robotic grasp actions.
\end{abstract}

\section{INTRODUCTION}

Two types of grasps are mainly considered for multifingered hands: power grasps, that use the whole surface of the hand to restrain the object, and precision grasps, that use only the fingertips to grasp the object. If friction is considered in the grasp analysis, the most frequent condition used to analyze the grasp is force closure (FC), i.e. that the forces applied by the fingers ensure the object immobility [1]. In general, given an object and a mechanical hand, there is more than one grasp that fulfills the force closure property. An optimal grasp is chosen using a quality measure, based on criteria such as geometrical considerations (e.g. the distance between the centroid of the grasp polygon and the center of mass of the object [2]), limits on the forces applied by the fingers on the object (e.g. the maximal disturbance resisted by the grasp [3]), task oriented quality measures [4], [5], or measures associated with the hand configuration [6]. A review of grasp quality measures is provided in [7].

By using different quality measures, a number of approaches have been developed for the synthesis of power [8], [9] and precision grasps [10], [11] for multifinger robotic hands. Human experience can also be used for interactively teaching the robot to perform good grasps, as humans successfully grasp almost any kind of object in an intuitive way. A person chooses a particular grasp using different criteria, learned through experience, that take into account factors such as comfort, task information and object-specific rules (such as grasping a mug by the handle). For teaching a robot to be successful in grasping tasks, it is not required to imitate the human movements, but to understand why the humans use their hands in the way they do. This knowledge can be

This work is supported by the DFG Center of Excellence EXC 277: Cognitive Interaction Technology (CITEC).

M.A. Roa (corresponding author) and C. Castellini are with the Institute of Robotics and Mechatronics, DLR (German Aerospace Center), D-82234 Weßling, Germany, \{maximo.roa, claudio.castellini\}@dlr.de.

R. Kõiva is with the Neuroinformatics Group, Center of Excellence Cognitive Interaction Technology, Bielefeld University, D-33615 Bielefeld, Germany, risto.koiva@uni-bielefeld.de.

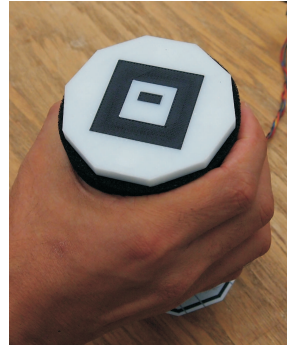

a)

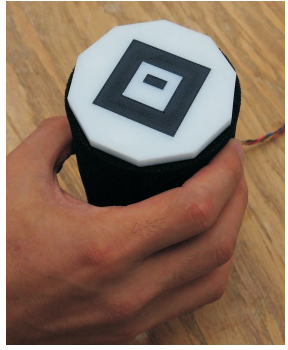

b)

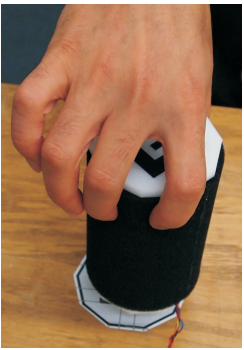

c)
Fig. 1. Examples of grasp types on the sensorized iObject: a) Side power grasp with 5 fingers; b) Side precision grasp with 5 fingers; c) Top precision grasp with 3 fingers.

exploited using for instance Programming by Demonstration to teach robots to grasp known and new objects in an effective way. A robotic hand can be instructed to imitate the hand preshape, grasp pose and approach direction shown by a human operator [12], although some a-priori knowledge of the limitations of the mechanical hands is required.

Work related to the study and evaluation of human grasps has focused on issues such as the relation between the object size and the aperture of the gripper [13], hand preshaping and fingertip trajectories [14], or force distribution among fingers during object manipulation [15], but just recently the application of concepts coming from the robotic world to the analysis of human grasps has gained attention. Human experience in grasping has been used for instance to guide a robotic arm and hand to grasp different objects, and lately to compare human-guided grasps to grasps obtained with a planner [16]. From that work, it was evident that humans prefer to align the palm to the object's principal axis. Moreover, the information coming from the task that the human wants to perform can be used to evaluate the quality of a robotic grasp intended for the same task [17].

This paper presents an initial study of different human grasps using analytical measures coming from the grasp community. An experiment was designed to acquire human grasp data using a sensorized object (iObject, [18]) equipped with tactile sensors that allow the acquisition of information on the spatial location of the contact points and magnitude of the forces applied to the object. The collected data were analyzed using different grasp quality measures, commonly used in robotic grasp planning, to compare the goodness of different types of grasps (Fig. 1), to evaluate how the grasp quality increases with the number of fingers and with the contact area involved in the grasp action, and to study the drawbacks of approximating a region of contact with simple contact points. Ultimately, the results of this study are 
useful to provide insight on how appropriate are the quality measures currently used in grasp planning for robot hands with respect to the evaluation of human grasps with the same criteria, and to help in the evolution of robotic grasping to achieve robustness closer to the human level.

The rest of the paper is organized as follows. Section II provides background on force closure grasps and Section III presents the grasp quality measures used in this study. Section IV describes the experimental setup and the test method used for gathering the data from the participants, and Section V presents the results of the data analysis using the selected quality measures. Finally, Section VI summarizes the paper and discusses on future work.

\section{BACKGROUND}

\section{A. Contact models}

The contact between the fingers and the object can be studied using mainly three models: frictionless and frictional contacts, and soft contacts [19]. Coulomb's friction model is used for frictional contacts, stating that to avoid slipping, the force $\boldsymbol{f}_{i}$ applied at position $\boldsymbol{p}_{\boldsymbol{i}}$ must lie inside the friction cone defined by $\boldsymbol{f}_{i}^{t} \leq \mu \boldsymbol{f}_{i}^{n}$, where $\mu$ is the friction coefficient and $\boldsymbol{f}_{i}^{t}$ and $\boldsymbol{f}_{i}^{n}$ are, respectively, the tangential and normal components of $\boldsymbol{f}_{i}$. The force $\boldsymbol{f}_{i}$ applied on the object at $\boldsymbol{p}_{i}$ generates a torque $\boldsymbol{\tau}_{i}=\boldsymbol{p}_{i} \times \boldsymbol{f}_{i}$ with respect to the center of mass $(C M) . f_{i}$ and $\tau_{i}$ are grouped together in the wrench vector $\boldsymbol{\omega}_{i}=\left(\boldsymbol{f}_{i} \boldsymbol{\tau}_{i}\right)^{T}$.

To ease the computational cost of dealing with the nonlinear friction cone, it is usually linearized using an $m$-side polyhedral convex cone. Thus, by representing the unitary vector along the $j$-th edge of the convex cone at the $i$-th contact with $\hat{\boldsymbol{n}}_{i j}$, the grasping force is given by:

$$
\boldsymbol{f}_{i}=\sum_{j=1}^{m} \alpha_{i j} \hat{\boldsymbol{n}}_{i j}, \quad \alpha_{i j} \geq 0
$$

If a unitary force is applied along an edge $\hat{\boldsymbol{n}}_{\boldsymbol{i j}}$ of the linearized cone, the corresponding wrench, $\boldsymbol{\omega}_{i j}$, is called a primitive wrench. A grasp is then defined by a set of contact points $C=\left\{\boldsymbol{p}_{1}, \ldots, \boldsymbol{p}_{n}\right\}$, and has an associated set $W=\left\{\boldsymbol{\omega}_{11}, \ldots, \boldsymbol{\omega}_{1 m}, \ldots, \boldsymbol{\omega}_{n 1}, \ldots, \boldsymbol{\omega}_{n m}\right\}$ of primitive wrenches.

The relation between the forces $f$ at the fingertips and the total wrench $\boldsymbol{\omega}$ applied on the object is given by the grasp matrix $G$, with $\boldsymbol{\omega}=G \boldsymbol{f}$ [19].

\section{B. Force closure}

A necessary and sufficient condition for the existence of a FC grasp is that the origin $O$ of the wrench space lies strictly inside the convex hull of $W$ [19], from now on represented as $C H(W)$. Several FC tests based on this condition have been proposed, for instance solving linear optimization problems [20], using collision checks [21], or with linear matrix inequalities [22].

The FC test implemented for this work verifies that the origin is inside $C H(W)$ by using as a reference point the centroid $P$ of the primitive contact wrenches in $W$, which
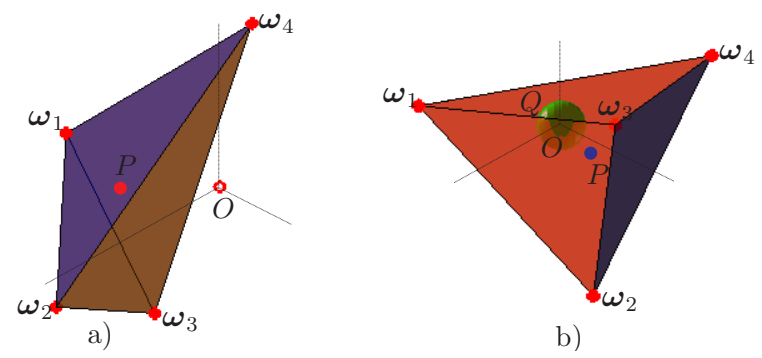

b)

Fig. 2. Force closure test: a) Non-FC grasp: the hyperplane formed by $\left\{\boldsymbol{\omega}_{2}, \boldsymbol{\omega}_{3}, \boldsymbol{\omega}_{4}\right\}$ leaves $P$ and $O$ in different half-spaces; b) FC grasp: all the supporting hyperplanes of $C H(W)$ leave $P$ and $O$ in the same half-space. The radius $Q$ of the largest inscribed sphere indicates the grasp quality.

is always an interior point of $C H(W)$. Then, for all the supporting hyperplanes $H$ of $C H(W)$, the points $O$ and $P$ should lie on the same halfspace [23]. Fig. 2 illustrates the concept with a FC grasp and a non-FC grasp for the 3-dimensional wrench space of a $2 \mathrm{D}$ object.

\section{GRASP QUALITY MEASURES}

A considerable number of grasp quality measures has been presented in the literature. However, all of them can be classified in mainly three groups: measures associated with the location of contact points on the object boundary, measures associated with the hand configuration, and hybrid measures that combine different basic criteria to get a combined index of quality [7]. Although some studies have compared the optimal grasps obtained according to different criteria for objects in 2D [24], [25] and 3D [26], the selection of the best criterion for each real case is not always trivial. To tackle this problem, this paper considers the most common measures used in grasp synthesis algorithms, and analyzes real grasp cases to verify what quality measure captures in a better way the intention of the human and describes more accurately the stability for different grasp types. For this purpose, we will compare the following quality measures:

1) Largest minimum resisted wrench: the most common measure used in grasp planning is the largest perturbation wrench that the grasp can resist independently of the perturbation direction [3]. Assuming, without loss of generality, that the maximum allowed grasping force is a unitary force along $\hat{\boldsymbol{n}}_{i j}$, this grasp quality is equivalent to the radius of the largest hypersphere centered on $O$ and fully contained in $\mathrm{CH}(W)$, i.e. it is the distance from $O$ to the closest facet of $C H(W)$ (Fig. 2b).

$$
Q_{1}=\min _{\boldsymbol{\omega} \in C H(W)}\|\boldsymbol{\omega}\|
$$

2) Volume of $C H(W)$ : another common measure is the volume of the convex hull of $W$, which is independent of the reference system used in the torque computation [26]. The maximization of the criterion leads to more robust grasps in general, although with the same volume a grasp could stand much less force than another one in a certain direction.

$$
Q_{2}=\operatorname{Volume}(C H(W))
$$


3) Distance between the centroid of the contact polygon/polyhedron and the center of mass of the object: the minimization of this distance reduces the effect of the inertial and gravitational forces on the grasp [27].

$$
Q_{3}=\operatorname{Dist}(C M, C)
$$

4) Volume of the grasp polyhedron: tries to maximize the volume of the convex hull of the contact points. It is a generalization of the idea of maximizing the area of a triangle for a tripod grasp [28].

$$
Q_{4}=\operatorname{Volume}\left(C H\left(\boldsymbol{p}_{1}, \boldsymbol{p}_{2}, \ldots, \boldsymbol{p}_{n}\right)\right)
$$

5) Minimum singular value of the grasp matrix $G$ : Larger values of this measure indicate that the grasp is farther from a singular grasp configuration (i.e. that the grasp loses the ability to withstand external wrenches in one or several directions) [6].

$$
Q_{5}=\sigma_{\min }(G)
$$

6) Grasp isotropy index of $G$ : looks for a uniform contribution of the contact forces to the total wrench applied on the object, i.e. it tries to balance the internal forces of the grasp [6]. The quality is defined as:

$$
Q_{6}=\frac{\sigma_{\min }(G)}{\sigma_{\max }(G)}
$$

\section{METHODS AND EXPERIMENTAL SETUP}

\section{A. Subjects}

Four adult healthy subjects, three males and one female (average age $33.7 \pm 4.9$ year) with no injuries or hand problems, gave the consent to participate in the study. All volunteers were right-handed, and all of them had an engineering background.

\section{B. Tactile sensor}

For capturing the contact locations and exerted pressure patterns of the participants during the grasping tasks of the experiment, an instrumented object, called iObject [18], was used. The iObject was developed for human and robotic manual interaction research; it has the size and shape of a $330 \mathrm{ml}$ soft drink can, and weighs $330 \mathrm{~g}$. The iObject includes, among other sensor modalities, 220 tactile sensor cells (tactels) on its decagon outer shell surface (Fig. 3). The tactile sensors, based on the resistive working principle [29], are ideal for capturing forces from the human hand due to their characteristic hyperbolic output curve, providing a finegrained signal near the low first contact range while still being able to output discriminating values for high finger forces. When rolled out flat, the tactile sensor measures $200 \times 115 \mathrm{~mm}$ and includes $20 \times 11$ tactels with a spatial resolution of $10 \mathrm{~mm}$. So far, the iObject has been used for a simple manipulation task [18]. This paper exploits the capabilities of such sensorized object for an extensive study of grasp qualities, as described in the following subsection.

For this experiment, two modifications were made to the original iObject design. First, the sensor material, the

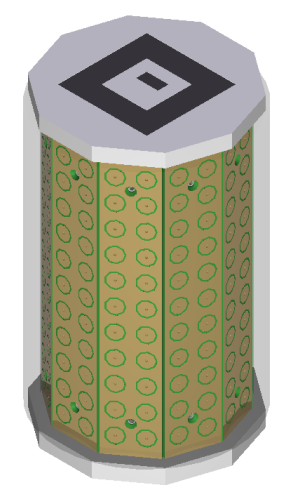

Fig. 3. iObject with 220 tactile sensor cells (tactels) on its decagon surface as seen through rendered translucent sensor material, black in reality.

conductive foam, was replaced with a softer version with higher electrical conductivity, shifting effectively the measurement range of the tactile sensor from the original 4 to $100 \mathrm{kPa}$ range into 1 to $30 \mathrm{kPa}$. Second, the default wireless Bluetooth connection was replaced by a cable based USB 2.0 connection to the PC to limit the jitter of the signal.

The tactile sensor of iObject was calibrated using a 3-axis numeric control table with an attached strain gauge sensor, sampling the tactels at numerous positions while exerting forces up to $20 \mathrm{~N}$. Although the measurement revealed minor variations in the output between tactels, caused by the resistivity inhomogeneity in the sensor foam, the relative change in the output was found univocal and usable for unitless grasp quality measurements.

\section{Procedure}

The aim of the experiment was to study various grasping strategies used when interacting with a common size, almostcylindrical object. The task presented to the participants was to grasp the iObject using 9 different grasp types. The types were power grasp on the side of the object, and side and top grasps using 5, 4, 3, and 2 fingertips (Fig. 1).

The participants were seated comfortably in front of a flat table (width $=80 \mathrm{~cm}$, height $=72 \mathrm{~cm}$ ), as shown in Fig. 4. All subjects were instructed to move the iObject on the table between two predefined positions, indicated with a drawing of the iObject silhouette, and with a separation of $30 \mathrm{~cm}$ between them. Visual stimulus from a PC display was used to specify the grasp type, while auditive stimulus from the same PC was used to trigger the movement. After each movement, the subject moved the hand to a resting position. Each participant repeated every grasp type 6 times, for a total of 54 recordings per subject. Tactile sensor data from the iObject was collected at 300 frames per second, and it was saved to a PC for a later offline processing. To gather information related to the subjective perception of grasp robustness, each subject was asked after the experiment to rate the robustness of the different types of grasps using a 7-point Likert scale. 


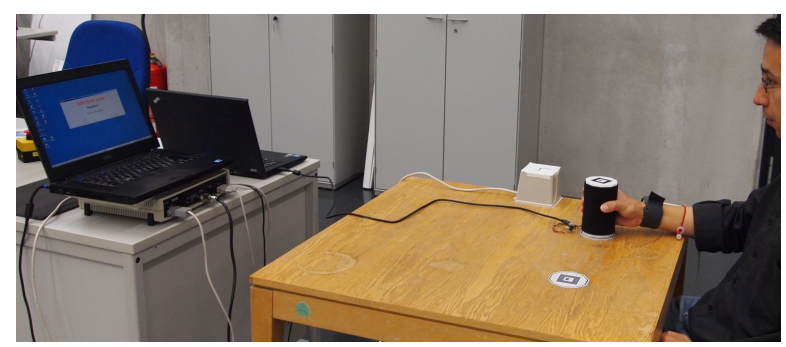

Fig. 4. Experiment setup for moving the iObject between two predefined positions using a grasp type indicated visually on the computer screen. The pace of the movement was signaled by an auditive stimulus.

TABLE I

SUBJECTIVE IMPRESSION OF GRASP ROBUSTNESS USING A 7-POINT LIKERT SCALE (THE HIGHER THE VALUE, THE BETTER THE GRASP ROBUSTNESS)

\begin{tabular}{|c|c|c|}
\hline Grasp type & Average & St. Dev. \\
\hline \hline Side, 5 fingers & 6.00 & 1.16 \\
\hline Side, 4 fingers & 5.29 & 1.38 \\
\hline Side, 3 fingers & 4.43 & 2.07 \\
\hline Side, 2 fingers & 2.71 & 1.70 \\
\hline Side, power grasp & 7.00 & 0.00 \\
\hline Top, 5 fingers & 5.29 & 1.11 \\
\hline Top, 4 fingers & 4.71 & 1.60 \\
\hline Top, 3 fingers & 3.57 & 1.27 \\
\hline Top, 2 fingers & 2.00 & 0.81 \\
\hline
\end{tabular}

\section{RESULTS}

The data generated with the experiment described in the previous section are analyzed using the quality measures presented in Section III. For the analysis, a friction coefficient of 0.4 , determined empirically, was used for the friction cone approximation at each contact point. As a first step, we use the quality measures $Q_{1}$ and $Q_{2}$ to compare the estimation of a grasp quality when the real contact area is approximated with one point per region of contact (which is a common assumption in grasp planning). A second comparison evaluates the goodness of different grasp types and the influence of the number of fingers involved in the grasping action, using the selected quality measures, and with a punctual approximation to each contact region.

The subjective impression of grasp robustness that the users report in our survey is summarized in Table I.

\section{A. Areas of contact vs. Punctual contacts}

The data coming from the sensorized object can be visualized as if the surface of the decagon were rolled out flat, as shown in Fig. 5a. The illuminated pixels indicate the tactels that receive some amount of pressure; the browner the color, the higher the pressure on a given tactel. The identification of the areas corresponding to each contact point is performed using a $k$-means clustering technique [30]. To apply this method, each tactel with pressure larger than zero was considered as an observation $z_{i}$, with $z_{i}=\left\{x_{i}, y_{i}, P_{i}\right\} \in \mathbb{R}^{3}$ containing the information on the location of each tactel on the developed surface $\left(x_{i}, y_{i}\right)$ and the pressure registered at each tactel $\left(P_{i}\right)$. To guarantee uniformity of the data, both

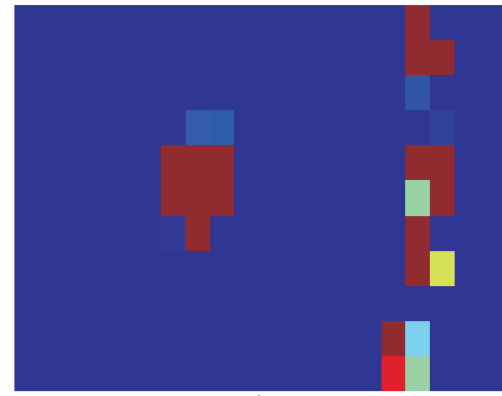

a)

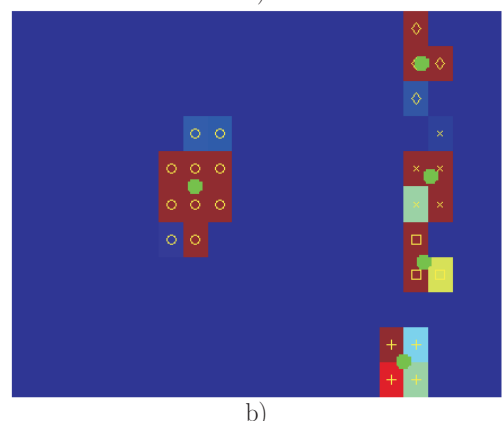

Fig. 5. Regions of contact vs. Punctual contact for a 5 finger grasp: a) Regions of contacts, as obtained from the iObject; b) Clusters corresponding to each contact region, and approximation to one contact point per contact region.

the coordinates and the pressure were normalized to lie in the interval $[0,1]$ for each time frame. Given the set of $m$ observations, the technique partitions the observations into $k$ sets $S$ such that they minimize the within-cluster sum of squares

$$
\min \sum_{l=1}^{k} \sum_{z_{j} \in S_{l}}\left\|z_{j}-\mu_{l}\right\|^{2}
$$

with $\mu_{l}$ the mean of the observations in $S_{l}$. The number $k$ of clusters must be known in advance. In the case of precision grasps, the number of clusters is equal to the number of fingers. However, for power grasps the identification of contact points is more complex, so we empirically chose 11 points to approximate a power grasp on the iObject. Fig. 5 shows the results for one example: Fig. 5a depicts the original data on the rolled surface, whereas Fig. 5b shows the result of the clustering (different symbols are used for each cluster), and highlights the corresponding centroids. The first two coordinates of the centroid $\mu_{l}$ for each cluster provide its location on the object surface, and therefore are used to approximate the region of contact with only one representative point for the following computations.

Each data set collected with the iObject was used to compute the grasp qualities $Q_{1}$ and $Q_{2}$ for both the contact region and the approximation with a contact point. These two measures were selected as they are the most common measures in grasp planning approaches. Fig. 6 shows the average and standard deviation of the results for each grasp type. Note that the underlying tendency is the same for both figures: the more contact points, the larger the quality for the considered types of grasps. However, the difference between 

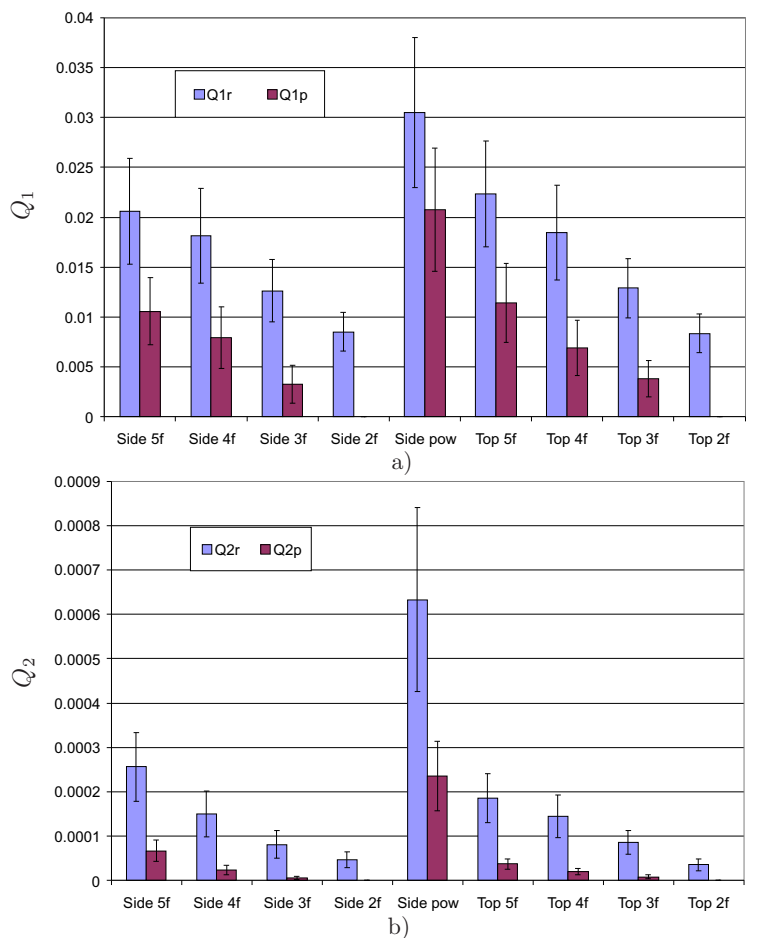

Fig. 6. Quality measures to compare the punctual approximation to contact regions: a) $Q_{1}$ for the region (r) and punctual approximation (p); b) $Q_{2}$ for the region (r) and punctual approximation (p). Error bars represent one standard deviation.

TABLE II

UNDERESTIMATION OF GRASP QUALITY $Q_{1}$ WITH THE PUNCTUAL APPROXIMATION TO CONTACT REGIONS.

\begin{tabular}{|c|c|c|c|}
\hline Grasp type & $Q_{1 r}$ & $Q_{1 p}$ & $\%$ Err \\
\hline \hline Side, 5 fingers & $2.06 \times 10^{-2}$ & $1.06 \times 10^{-2}$ & 48.8 \\
\hline Side, 4 fingers & $1.81 \times 10^{-2}$ & $7.92 \times 10^{-3}$ & 56.3 \\
\hline Side, 3 fingers & $1.26 \times 10^{-2}$ & $3.27 \times 10^{-3}$ & 74.1 \\
\hline Side, 2 fingers & $8.52 \times 10^{-3}$ & 0 & 100 \\
\hline Side, power grasp & $3.05 \times 10^{-2}$ & $2.07 \times 10^{-2}$ & 32.0 \\
\hline Top, 5 fingers & $2,23 \times 10^{-2}$ & $1.14 \times 10^{-2}$ & 48.8 \\
\hline Top, 4 fingers & $1.85 \times 10^{-2}$ & $6.91 \times 10^{-3}$ & 62.6 \\
\hline Top, 3 fingers & $1.29 \times 10^{-2}$ & $3.80 \times 10^{-3}$ & 70.5 \\
\hline Top, 2 fingers & $8.35 \times 10^{-3}$ & 0 & 100 \\
\hline
\end{tabular}

power and precision grasps is much larger when the punctual approximation is used. There is no big difference in quality between top and side precision grasps performed with the same number of fingers when the contact region is used in the computations (except perhaps for the case of 5 finger grasps). However, using punctual contacts it seems that the top grasps have more quality than the side grasps with both quality measures, which is unexpected, given that for the users it seems more intuitive to grasp the object by the side, and grasping it by the top gives a subjective impression of a higher instability (Table I). For the two finger grasps the quality of the punctual approximation was zero, i.e. the centroid of the regions does not represent accurately the contact state of the whole object.

Tables II and III summarize the results of the average
TABLE III

UNDERESTIMATION OF GRASP QUALITY $Q_{2}$ WITH THE PUNCTUAL APPROXIMATION TO CONTACT REGIONS.

\begin{tabular}{|c|c|c|c|}
\hline Grasp type & $Q_{2 r}$ & $Q_{2 p}$ & $\%$ Err \\
\hline \hline Side, 5 fingers & $2.56 \times 10^{-4}$ & $6.66 \times 10^{-5}$ & 74.0 \\
\hline Side, 4 fingers & $1.50 \times 10^{-4}$ & $2.38 \times 10^{-5}$ & 84.1 \\
\hline Side, 3 fingers & $8.14 \times 10^{-5}$ & $6.19 \times 10^{-6}$ & 92.4 \\
\hline Side, 2 fingers & $4.65 \times 10^{-5}$ & 0 & 100 \\
\hline Side, power grasp & $6.33 \times 10^{-4}$ & $2.36 \times 10^{-4}$ & 62.7 \\
\hline Top, 5 fingers & $1.86 \times 10^{-4}$ & $3.71 \times 10^{-5}$ & 80.1 \\
\hline Top, 4 fingers & $1.44 \times 10^{-4}$ & $1.96 \times 10^{-5}$ & 86.5 \\
\hline Top, 3 fingers & $8.64 \times 10^{-5}$ & $8.00 \times 10^{-6}$ & 90.7 \\
\hline Top, 2 fingers & $3.56 \times 10^{-5}$ & 0 & 100 \\
\hline
\end{tabular}

quality obtained for the different grasp types, and includes the percentage of underestimation induced in the grasp quality computation when the contact regions are approximated with only one contact point. Note that the errors are much worse when $Q_{2}$ is used; therefore, $Q_{1}$ seems to be a more appropriate measure to use when the grasps are analyzed using punctual contacts.

Fig. 7 shows a typical evolution in time of the quality $Q_{1}$ both for the region and punctual contacts, for the data collected with one movement of the iObject between the reference positions using a 5 finger side grasp. The evolution from zero to top quality occurs in approx. $0.1 \mathrm{~s}$ for the punctual approximation, but it takes about $0.5 \mathrm{~s}$ when the full contact region is considered.

\section{B. Comparison of different grasp types}

Fig. 8a shows the results for $Q_{3}$, the distance between the centroid of the contact points and the $C M$ of the object. Note first that this measure is not affected by the quality of the punctual approximation for the two finger grasp. This measure should be low to ensure a good grasp. For all the side grasps and for the power grasp, the average value obtained is very similar, so the measure is not a good discriminant in these cases. For the top grasps the measure is higher (i.e. worse quality) for a lower number of fingers, but this effect of the number of fingers on the grasp quality is not evident for lateral grasps.

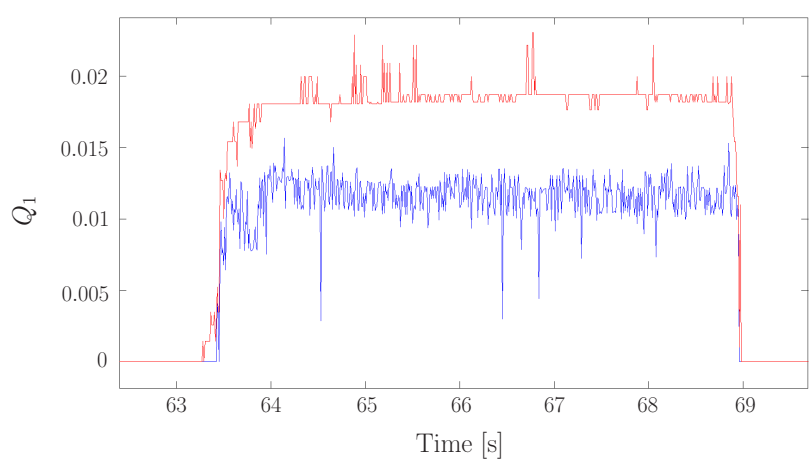

Fig. 7. Evaluation in time of $Q_{1}$ for the region (red line) and the punctual contact (blue line), for the data collected for one movement of the iObject with a 5 finger side grasp. 

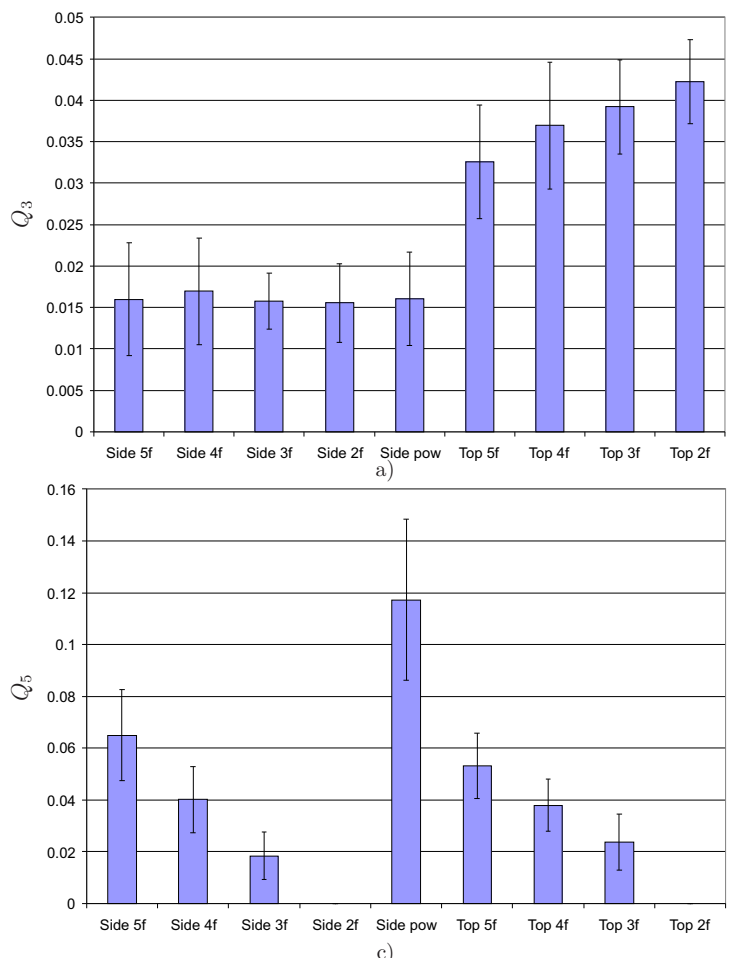
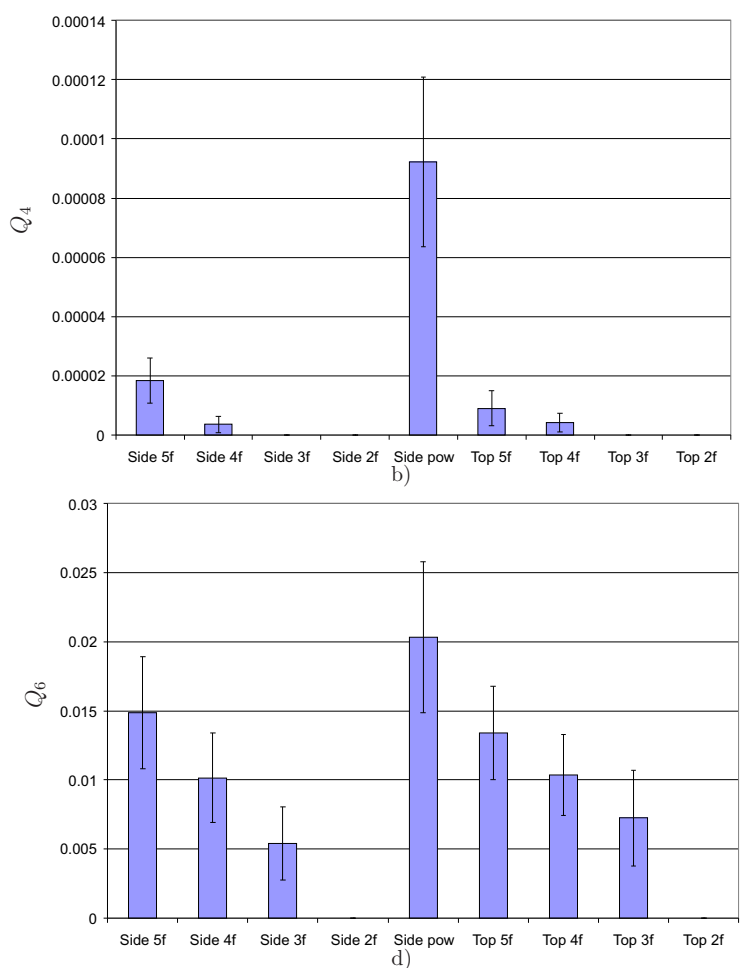

Fig. 8. Quality measures to compare different grasp types: a) $Q_{3}$; b) $Q_{4}$; c) $Q_{5}$; d) $Q_{6}$. Error bars represent one standard deviation. Note that a good grasp would minimize the value of $Q_{3}$, or maximize the value of $Q_{4}, Q_{5}$, and $Q_{6}$.

The results for $Q_{4}$ are shown in Fig. 8b. A good grasp according to this measure tends to maximize the volume of the contact polyhedron (which is not defined for 2 and 3 fingers). In this case, the power grasp has considerably higher quality with respect to the fingertip grasps. For 5 fingers, the side grasp looks better than the top one. However, for 4 fingers the measure does not indicate any difference. The practical utility of this measure seems to be very low. Its application should be restricted to the case of 3 finger grasps, where the area of the triangle formed by the contacts must be maximized [31] (the area of such polygon was not computed in this analysis).

Fig. $8 \mathrm{c}$ and $8 \mathrm{~d}$ show the comparison of the grasp qualities $Q_{5}$ and $Q_{6}$, i.e. the minimum singular value and the isotropy index, both computed for the grasp matrix $G$. In both cases, the larger the value the better the grasp. The behavior of both measures is very similar: the power grasp gets the maximum values, and for the fingertip grasps, the more fingers the better the quality. Also, the measures do not indicate a significant difference between side and top grasps, except for the 5 finger case.

\section{DISCUSSION AND FUTURE WORKS}

This paper has presented initial results of the comparative analysis of several grasp quality measures, initially defined in the robotic grasp literature, applied to the evaluation of human grasps. The data were collected using a sensorized object that provides information on the location and pressure applied at the contact points on the object.

As a first insight gained with this experiment, we note that the approximation of the real contact regions with punctual contact models greatly underestimates the grasp qualities. It is also worth noting that this effect is more important for the human hand, which has highly compliant fingertips and deformable skin, than for typical robotic hands, with fingertips usually made of harder materials, for which the fingertip deformation is not so high. In particular, for the measure $Q_{2}$ the degradation of the quality estimation for the punctual approximation is very high, while for $Q_{1}$ such underestimation is less critical. Also, due to the resolution of the iObject, the punctual approximation for the case of 2 finger grasps is not good, as the estimated contact point using the $k$-means algorithm does not lead to a force closure grasp.

A second goal of this study was the comparison of the behavior for several quality measures for the evaluation of different grasp types; the results were contrasted with the subjective experience of the participants in the experiment. For them, using the same number of fingers, the top grasps felt weaker than the lateral grasps. In that sense, the measure $Q_{3}$ that quantifies the distance between the $C M$ and the centroid of the contact points seems to agree more with the impression of the participants and with the results from [16], in the sense that the subjects preferred grasps aligned with the principal axis of the object. However, such measure does not indicate a large influence of the number of fingers in the grasp quality in the case of side grasps. The practical utility of the measure $Q_{4}$ is very limited, and should be restricted to the case of 3 finger grasps, where the maximization of the area of the polygon formed by the 
TABLE IV

CORRELATION INDEX BETWEEN THE ANALYTICAL MEASURES AND THE SUBJECTIVE PERCEPTION OF GRASP ROBUSTNESS.

\begin{tabular}{|c|c|c|c|c|c|c|c|}
\hline Q1r & Q1p & Q2r & Q2p & Q3 & Q4 & Q5 & Q6 \\
\hline \hline 0.94 & 0.93 & 0.82 & 0.75 & 0.49 & 0.92 & 0.93 & 0.92 \\
\hline
\end{tabular}

contact points leads to more robust grasps. The measures $Q_{5}$ and $Q_{6}$, which only take into account algebraic properties of the grasp matrix $G$, seem to have the same behavior, i.e. they provide basically the same information. The behavior of these measures also indicates clearly that the more fingers involved in the grasping action, the better the quality (which is not so clear with the measure $Q_{3}$ for instance).

To provide a better assessment on the relation between the subjective perception of the participants and the analytical results, Table IV indicates the Pearson product-moment correlation coefficient between the quality measures and the results of the perception survey. The coefficient was computed taking into account only the grasps where each quality measure was valid (for instance, the case of 2 finger grasps is not considered for $Q_{5}$ and $Q_{6}$ ). Note that the best correlation is obtained for $Q_{1} r$. Despite the underestimation of grasp quality that the punctual approximation to the contact region creates, the correlation index was also very high for the case of $Q_{1} p$. Other quality measures with high correlation coefficients were $Q_{4}, Q_{5}$, and $Q_{6}$, although in the case of $Q_{4}$ the measure was only valid for 5 and 4 finger grasps, and in the cases of $Q_{5}$ and $Q_{6}$ the resolution of the sensorized object was not enough to create a good punctual approximation to the contact region for the 2 finger case. In the case of $Q_{3}$, the low index reflects the problems that the measure has to differentiate the quality for lateral grasps with different number of fingers.

For an improved grasp quality assessment, a combined index that mixes the results of several quality measures could be an option worth being explored. Such combined index could also include quality measures that take into account the posture of the grasping hand, not evaluated in the present experiment. As another future work, we want to include task oriented measures in a similar study. Besides that, the development of an iObject with a higher resolution will provide us with more detailed information on the real contacts on the object.

\section{REFERENCES}

[1] A. Bicchi, "On the closure properties of robotic grasping," Int. J. Robotics Research, vol. 14, no. 4, pp. 319-344, 1995.

[2] J. Ponce and B. Faverjon, "On computing three-finger force-closure grasps of polygonal objects," IEEE Trans. Robotics and Automation, vol. 11 , no. 6, pp. 868-881, 1995.

[3] C. Ferrari and J. Canny, "Planning optimal grasps," in IEEE Int. Conf. on Robotics and Automation, 1992, pp. 2290-2295.

[4] Z. Li and S. Sastry, "Task-oriented optimal grasping by multifingered robotic hands," IEEE J. Robotics and Automation, vol. 4, no. 1, pp. 32-44, 1988

[5] C. Borst, M. Fischer, and G. Hirzinger, "Grasp planning: How to choose a suitable task wrench space," in IEEE Int. Conf. on Robotics and Automation, 2004, pp. 319-325.
[6] K. Shimoga, "Robot grasp synthesis algorithms: A survey," Int. J. Robotics Research, vol. 15, no. 3, pp. 230-266, 1996.

[7] R. Suarez, M. Roa, and J. Cornella, "Grasp quality measures," Technical University of Catalunya, Tech. Rep. IOC-DT-P-2006-10, 2006.

[8] A. Miller, S. Knoop, H. Christensen, and P. Allen, "Automatic grasp planning using shape primitives," in IEEE Int. Conf. on Robotics and Automation, 2003, pp. 1824-1829.

[9] C. Goldfeder, P. Allen, C. Lackner, and R. Pelossof, "Grasp planning via decomposition trees," in IEEE Int. Conf. on Robotics and Automation, 2007, pp. 4679-4684.

[10] Y. Liu, M. Lam, and D. Ding, "A complete and efficient algorithm for searching 3-D form closure grasps in the discrete domain," IEEE Trans. Robotics, vol. 20, no. 5, pp. 805-816, 2004.

[11] M. Roa and R. Suarez, "Finding locally optimum force-closure grasps," J. Robotics and Computer Integrated Manufacturing, vol. 25, no. 3, pp. 536-544, 2009.

[12] S. Ekvall and D. Kragic, "Learning and evaluation of the approach vector for automatic grasp generation and planning," in IEEE Int. Conf. on Robotics and Automation, 2007, pp. 4715-4720.

[13] R. H. Cuijpers, J. B. Smeets, and E. Brenner, "On the relation between object shape and grasping kinematics," J. Neurophysiology, vol. 91, pp. 2598-2606, 2004

[14] T. Supuk, T. Kodek, and T. Bajd, "Estimation of hand preshaping during human grasping," Medical Engineering \& Physics, vol. 27, pp. 790-797, 2005.

[15] S. Li, "Perception of individual finger forces during multi-finger force production tasks," Neuroscience Letters, vol. 409, no. 3, pp. 239-243, 2006

[16] R. Balasubramanian, L. Xu, P. D. Brook, J. R. Smith, and Y. Matsuoka, "Human-guided grasp measures improve grasp robustness on physical robot," in IEEE Int. Conf. on Robotics and Automation, 2010, pp. 2294-2301.

[17] J. Aleotti and S. Caselli, "Interactive teaching of task-oriented robot grasps," Robotics and Autonomous Systems, vol. 58, pp. 539-550, 2010.

[18] R. Kõiva, R. Haschke, and H. Ritter, "Development of an intelligent object for grasp and manipulation research," in IEEE Int. Conf. Advanced Robotics - ICAR, 2011, pp. 204-210.

[19] R. Murray, Z. Li, and S. Sastry, A Mathematical Introduction to Robotic Manipulation. Boca Raton, Florida: CRC Press, 1994.

[20] Y. Liu, "Qualitative test and force optimization of 3-D frictional formclosure grasps using linear programming," IEEE Trans. Robotics and Automation, vol. 15, no. 1, pp. 163-173, 1999.

[21] X. Zhu, H. Ding, and S. Tso, "A pseudodistance function and its applications," IEEE Trans. Robotics and Automation, vol. 20, no. 2, pp. 344-352, 2004

[22] L. Han, J. Trinkle, and Z. Li, "Grasp analysis as linear matrix inequality problems," IEEE Trans. Robotics and Automation, vol. 16 , no. 6, pp. 663-674, 2000.

[23] M. Roa and R. Suarez, "Computation of independent contact regions for grasping 3-D objects," IEEE Trans. Robotics, vol. 25, no. 4, pp. 839-850, 2009.

[24] G. Bone and Y. Du, "Multi-metric comparison of optimal 2D grasp planning algorithms," in IEEE Int. Conf. on Robotics and Automation, 2001, pp. 3061-3066.

[25] A. Morales, P. Sanz, A. del Pobil, and A. Fagg, "An experiment in constraining vision-based finger contact selection with gripper geometry," in IEEE/RSJ Int. Conf. on Intelligent Robots and Systems, 2002 , pp. $1693-1698$

[26] A. Miller and P. Allen, "Examples of 3D grasp quality computations," in IEEE Int. Conf. on Robotics and Automation, 1999, pp. 1240-1246.

[27] J. Ponce, S. Sullivan, A. Sudsang, J. Boissonat, and J. Merlet, "On computing four-finger equilibrium and force-closure grasps of polyhedral objects," Int. J. Robotics Research, vol. 16, no. 1, pp. 1135, 1997.

[28] E. Chinellato, R. Fisher, A. Morales, and A. del Pobil, "Ranking planar grasp configurations for a three-finger hand," in IEEE Int. Conf. on Robotics and Automation, 2003, pp. 1133-1138.

[29] K. Weiss and H. Worn, "The working principle of resistive tactile sensor cells," in IEEE Int. Conf. Mechatronics and Automation - ICMA 2005, pp. 471-476.

[30] G. Seber, Multivariate Observations. John Wiley \& Sons, 2004.

[31] B. Mirtich and J. Canny, "Easily computable optimum grasps in 2D and 3D," in IEEE Int. Conf. on Robotics and Automation, 1994, pp. 739-747. 